\title{
Fast tetrahedron-tetrahedron overlap algorithm
}

\author{
F. Ganovelli, F. Ponchio and C. Rocchini
}

August 11, 2002

\begin{abstract}
We present an algorithm to test two tetrahedra for overlap. The algorithm is based on a dimension reduction technique that allows to apply the Separating Axis Theorem avoiding part of the computation needed to perform the Separating Axis Test. Source code is available online.
\end{abstract}

\section{Introduction}

The algorithms to test overlap between two convex polyhedra made of few faces [4] work trying to find a plane that separates the vertices of the two polytopes. The Separating Axis Theorem $[3,1,2]$ states that if two convex polytopes $a$ and $b$ do not overlap, then there exists an axis on which their projections do not overlap and such axis satisfies one of the following:

1. it is orthogonal to a face of one of the two polytopes;

2. it is orthogonal to an edge for each of the two polytopes;

The application of SAT consists of testing if the plane lying on the face of a polytope separates the two of them. If this is not the case, we should find out if there is a pair of edges, one for each polytope, such that the projections of the two polytopes on $\bar{e}_{a} \times \bar{e}_{b}$ do not overlap, where $\bar{e}_{a}\left(\bar{e}_{b}\right)$ is the vector obtained as the difference between the extremes of the edge $e_{a}\left(e_{b}\right)$.

The cost in terms of floating point operations of this procedure over a tetrahedron is easily determined as follows:

1. The cost of determining if there is a separating axis orthogonal to a face is: 18 multiplications, 8 summations, 21 subtractions, and 4 comparisons;

2. The cost of determining if there is a separating axis orthogonal to a pair of edges is: 30 floating point multiplications, 19 summations, 6 subtractions and 16 comparisons.

Since the cost of the test 2) is higher and the number of times it must be done is quadratic on the number of edges, for standard SAT is generally more efficient to do test 1) first for all faces of one polyhedron, and then do test (2) for all 
pairs of edges.

The algorithm we present reduces the cost of the test 2) to 2 multiplications, 1 subtraction and 1 comparison, and so allows to change the order in which tests are performed to optimize the final performance. Source code is available at the web site listed at the end of this paper. The rest of the paper proceeds as follows: Section 2 is the core part of the paper and it describes our idea for a smart implementation of the Separating Axis Test, along with Section 3 that contains the pseudocode of the whole algorithm. Results are reported in Section 4 and a final discussion in Section 5.

\section{Our approach}

The main advantage of our approach, referred as GPR algorithm in the following, is that it does not involve explicitly testing pairs of edges to find a separating axis. Instead, reusing some of the quantities computed to find a separating plane lying on a face, we give a way to discard the possibility that a separating plane lying on a edge exists, at the small cost of 2 scalar multiplications and 1 summation (i.e. a bidimensional vector product).

The approach is based on the following observation: lets $e$ be an edge, $f_{0}, f_{1}$ faces containing that edge, $p_{0}, p_{1}$ half-planes extending those faces, and $W$ be the resulting wedge (which contains the tetrahedron), then if the other tetrahedron intersects $W$ there cannot be a separating plane containing $e$.

The algorithm is based on three kind of tests: Face $(i)$, Edge $(f 0, f 1)$, and PointInside() explained below.

Face (i)

This function implements test (1) described in Section 1: it returns true iff the plane $P_{i}$ containing the face $i$ is a separating plane (or, equivalently, if the face normal is a separating axis). Firstly, the normal to the face $i$ pointing outwards the tetrahedra is computed as the vector product of two vectors oriented like two edges of the face :

$$
n(i)=\overline{e_{0}} \times \overline{e_{1}}
$$

Then each vertex of the other tetrahedron is tested to see if it belongs to the halfspace $h_{i}$ defined by the plane $P_{i}$ and the normal $n_{i}$.

$$
\text { if }\left(\left(\operatorname{coord}[i][j]=\left(n(i) \cdot\left(b_{j}-a_{0}\right)\right)>0\right) \operatorname{masks}[i] \mid=2^{j}\right.
$$

If the test succeeds for all of the four vertices, then the plane $P_{i}$ is a separating plane and the function returns true. Note that we store the results of this test: masks $[i]$ stores in which the side of the plane each vertex of the other tetrahedron is included (see Figure 1) and coord $[i][j]$ stores the result of the dot product. 


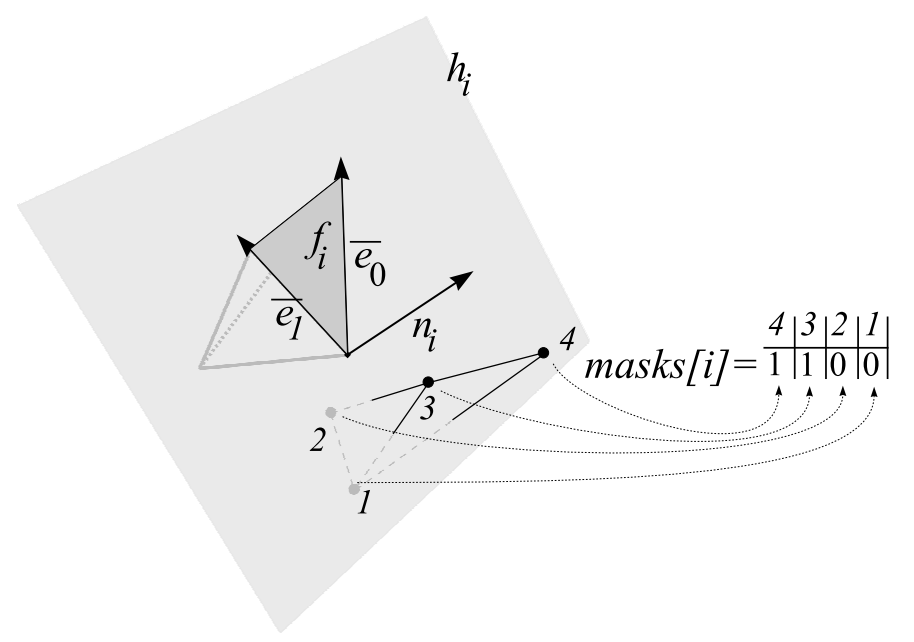

Figure 1: The vertices $1 \ldots 4$ are tested to see if they are contained in the halfspace $h_{i}$ and the result is stored in masks $[i]$ as a bit

Edge $(f 0, f 1)$

This function implements test (2) described in Section 1 returns true iff there exists a separating plane containing the edge $e$ shared by the faces $f 0$ and $f 1$ of the tetrahedron $a$.

Figure 2.(a) shows shows the application of the test for the edge $e$ shared by faces $f 0$ and $f 1$ of tetrahedron $a$. Lets $P_{w}$ be a plane orthogonal to $e$ : a separating plane containing $e$ exists iff there exists a line passing through point $e^{\prime}$ (projection of $e$ on $P_{w}$ ) separating the projections of the two tetrahedra $a$ and $b$.

This observation allows us to reduce dimension of the problem by 1 , projecting everything on $P_{w}$ (see Figure 2.(b)).

We can express points of $P_{w}$ in a coordinate system centered in $e^{\prime}$ with axes $n_{0}$ and $n_{1}$ orthogonal respectively to $f_{0}$ and $f_{1}$ (see Figure 2.(c)).

Coordinates of the projection $b_{j}{ }^{\prime}$ of a vertex of $b$ will be $\left(b_{j} \cdot n_{0}, b_{j} \cdot n_{1}\right)$ and we find them conveniently stored in $\operatorname{coord}[i][j], i=f_{0}, f_{1}$ and $j=1 \ldots 4$.

Furthermore, the values masks $[j], j=1 \ldots 4$ tell us in which quadrant the ver$\operatorname{tex} b_{j}{ }^{\prime}$ is.

Now all we need to test is that no point of $b^{\prime}$ lies in the quadrant $(-,-)$, as this will grant the existence of a separating line (and viceversa). It will be enough to test that no vertex $b_{i}$ lies in $(-,-)$ and that no edge $b_{i}{ }^{\prime}, b_{j}{ }^{\prime}$ intersect $(-,-)$.

Since edges having one or two extremes in $(+,+)$ cannot intersect $(-,-)$, we 
can use the masks computed in Face $A(f 0)$ and Face $A(f 1)$ to perform a quick rejection test:

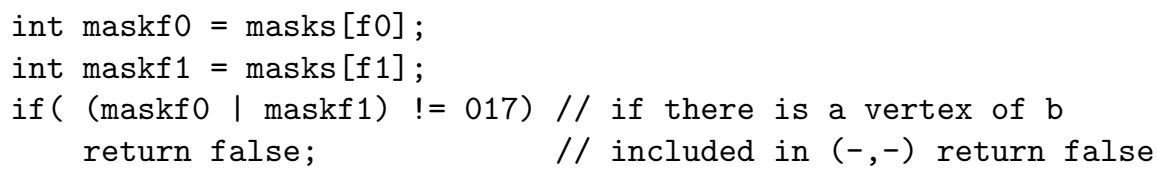

Next, we exclude vertices in $(+,+)$ :

maskf0 \& $=(\operatorname{maskf0}-\operatorname{maskf} 1) ; / /$ exclude the vertices in $(+,+)$

$\operatorname{maskf1} \&=(\operatorname{maskf0} \sim \operatorname{maskf} 1)$;

For the edges having an extreme in $(-,+)$ and the other in $(+,-)$ the test is a mere bidimensional vector product $b_{i}{ }^{\prime} \times b_{j}{ }^{\prime}$ (see Figure 2.(c)). For edge $0--1$ of $b$, we test:

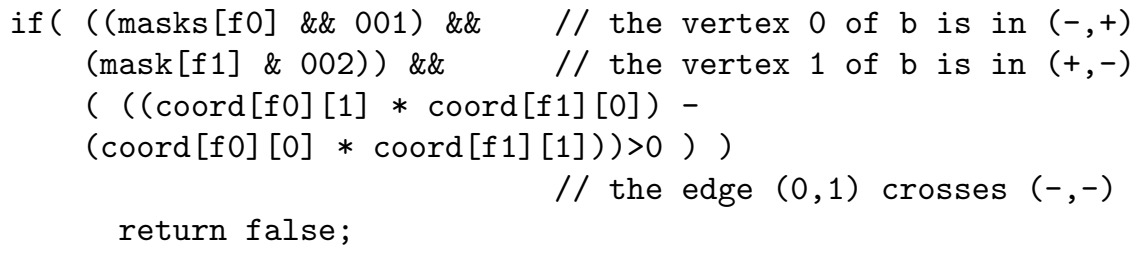

and the other edges of $b, 0-2,0-3,1-2,1-3$ and $2-3$, are tested similarly.

PointInside ()

PointInside() returns true if at least a vertex of of $b$ is inside the tetrahedron $a$. It is performed after the tests on the faces have computed the values of the array masks and it consists of the following row:

$$
\text { if (masks[0] | masks[1] | masks[2] | masks[3] != 1111) return true; }
$$

If the test succeeds than at least a bit is zero in every masks, that means that the corresponding vertex is inside all of the four halfspaces.

\section{Overall algorithm}

The algorithm is straightforward:

bool algorithm tet_a_tet(Tetrahedron A, Tetrahedron B)

\{

if (FaceA(0)) then return false 

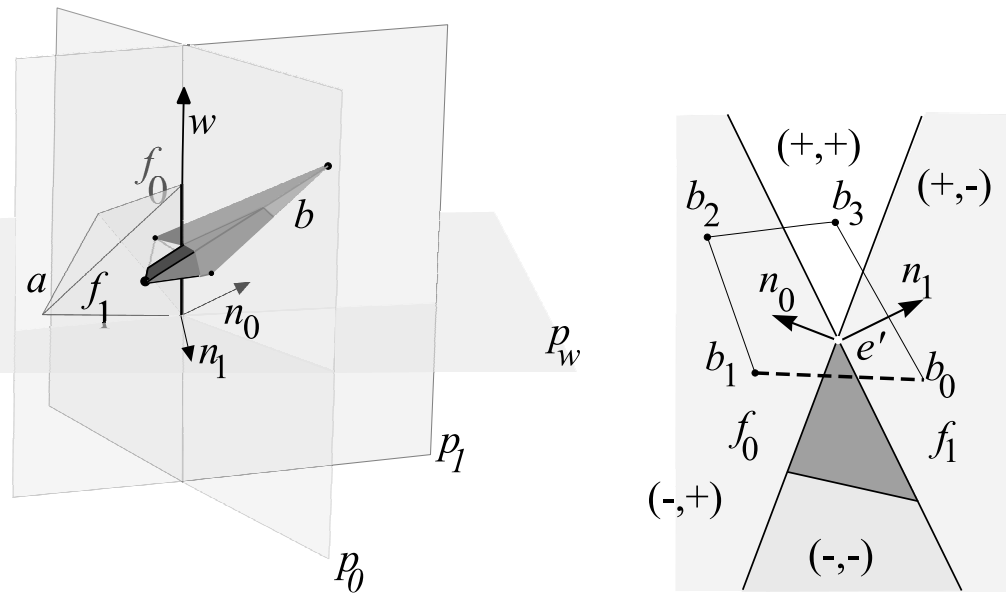

(a)

(b)

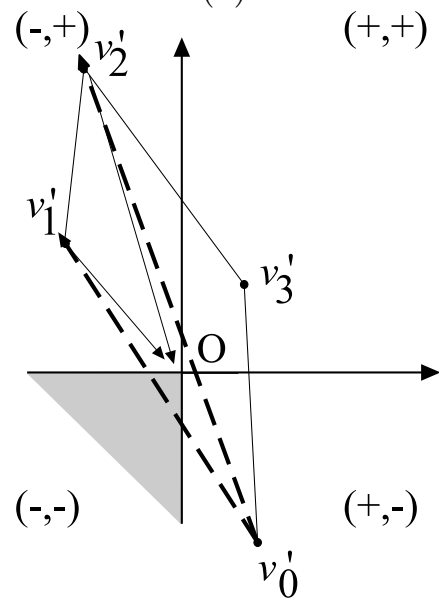

(c)

Figure 2: (a) If the tetrahedra $a$ and $b$ overlap, so do their projections on $P_{w}(\mathrm{~b})$ the same scene as (a) seen from a point along the $w$ axis; (c) Since $b_{0}^{\prime} \times b_{2}^{\prime}>0$, $b_{0}^{\prime} \rightarrow b_{2}^{\prime} \rightarrow O$ is a left turn and $\overline{b_{0}^{\prime} b_{2}^{\prime}}$ does not intersect the quadrant (-,-). Conversely, $b_{0}^{\prime} \times b_{1}^{\prime}<0, v_{0}^{\prime} \rightarrow v_{1}^{\prime} \rightarrow O$ is a right turn and $\overline{b_{0}^{\prime} b_{1}^{\prime}}$ intersects the quadrant $(-,-)$ 


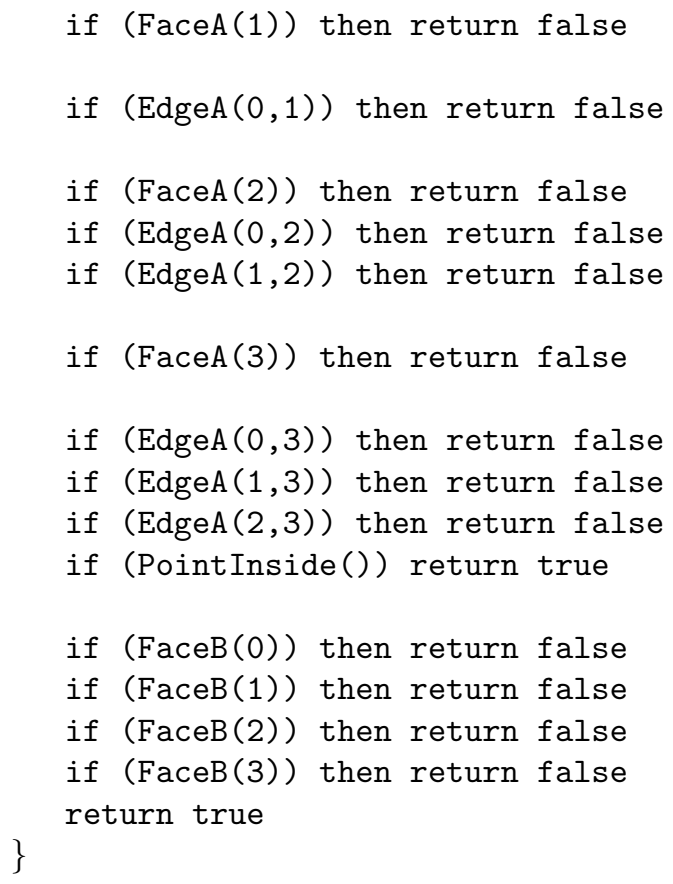

Note that the $\operatorname{EdgeA}(i, j)$ is performed as soon as FaceA( $i)$ and FaceA( $j$ ) have been performed, so that the values of coords and masks for $i$ and $j$ are known. Also note that no tests on the edges of the tetrahedron $b$ are done, because if there existed a separating axis $\bar{e}_{a} \times \bar{e}_{b}$, then there would exist a separating plane laying on an edge of $a$ and after the last EdgeA() it is already known that there cannot be such a plane.

The test PointInside() performs a trivial accept based on already-computed values, to avoid the remaining computations; it test if at least one of the vertices of tetrahedron $b$ is inside tetrahedron $a$ and could be omitted with no effect on the correctness.

\section{Results}

We compare our algorithm with the standard Separating Axis Test algorithm, where the tests on the separating axis orthogonal to a pair of edges are left after the tests on the faces.

The test-bed consists of testing overlap of two tetrahedra randomly generated. The random generation of a tetrahedron is done by generating its four vertices in a unit cube. In the first experiment such a cube is the same for both tetrahedra; in the second the two cubes share a face and in the third one are far apart. This subdivision is done in order to test our algorithm in different circumstances: when the tetrahedra overlap, when they do not overlap but are close to each other and when they are far away. Table 1 reports the results of 


\begin{tabular}{|l|c|c|c|}
\hline & set 1 & set 2 & set 3 \\
\hline time GPR & 29.1 & 17.1 & 13.4 \\
\hline time SAT & 101.2 & 19.4 & 13.0 \\
\hline \#faces GPR & 2.41 & 2.14 & 1.74 \\
\hline \#faces SAT & 3.34 & 2.62 & 1.75 \\
\hline
\end{tabular}

Table 1: Results: the first two rows report the number of milliseconds to perform $10^{4}$ overlap tests; the second two rows the average number of face tested. In set 1 , the tetrahedra are likely to overlap, in set 2 they are close but on overlapping, and in set 3 they are far away from each other.

these tests in milliseconds over $10^{4}$ pairs of tetrahedra to test (Intel Pentium IV $1 \mathrm{Ghz}$ ). You can see how GPR algorithm is much faster when the two tetrahedra are likely to collide, while its performance is substantially the same as SAT algorithm in the other conditions. This because if the tetrahedra are far apart, then the separating plane will (almost) always lay on a face and it will be found without testing pairs of edges. As a consequence the two algorithms perform the same operations with the difference that GPR pays the overhead of storing the result of the tests on the faces. On the contrary, when the tetrahedra are close to each other, the GPR algorithm tests less faces of SAT, thanks to the fact that testing for separating planes lying on edges is almost negligible and it can be done earlier in the algorithm.

\section{Discussion}

This paper gives a fast overlap test between two tetrahedra. No divisions are involved and the only errors that can arise are the ones given by scalar products and subtractions. If the first tetrahedron is flat, i.e. its four vertices lay on a plane, then it is possible to get a wrong answer. In particular the coordinate system given by the normals of two faces with opposite orientation is degenerate and any point on a line perpendicular to the axes is transformed on the same point in the affine coordinate system (see Figure 3.(a)). The more, all of the points will be on the same straight line passing through the origin of the affine coordinate system (see Figure 3.(b)). This means that no meaningful answer is given in this case.

Finally we stress that the GPR algorithm is mainly based on the dimensional reduction technique that offers the way to save operations at the price of storing some more data. The idea we presented can be used as it is with general convex polygons, as long as the overhead caused by accessing memory to store such data is not greater than the time saved. This of course depends on the hardware architecture, so we cannot say a priori the number of faces the polygons must have in order to be convenient to use our algorithm, but we empirically estimate such number to be under 10 . 


\section{Web Information}

Source code for the GPR algorithm is available at http://www.acm.org/jgt/papers/GanovelliPonchioRocchini02

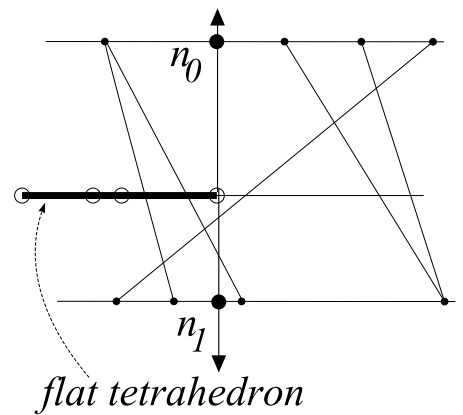

(a)

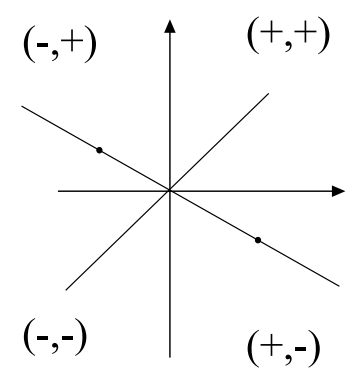

(b)

Figure 3: (a) Degenerate case: all of the points project on the same on the affine coordinates system (b) The projection of any edge in affine coordinate system pass through the origin.

\section{References}

[1] Tomas Akenine-Moller. Fast 3d triangle-box overlap testing. JGT, 2(2):2530, 1997.

[2] Van Den Bergen G. Efficient collision detection of complex deformable models using aabb trees. Journal of Graphics Tools, 2(4):1-13, 1998.

[3] S. Gottschalk, M. Lin, and D. Manocha. OBBTree: A hierarchical structure for rapid interference detection. Computer Graphics, 30(Annual Conference Series):171-180, 1996.

[4] Rich Rabbitz. Fast collision detection of moving convex polyhedra. In Paul Heckbert, editor, Graphics Gems IV, pages 83-109. Academic Press, Boston, 1994. 